\title{
La beauté et l'enfer se disent à voix basse : dire le mal et témoigner dans Kaputt
}

La bellezza e l'inferno si dicono a voce bassa: dire il male e testimoniare in Kaputt

Beauty and the Inferno "in a Low Voice": Voicing Evil and Bearing Witness in Malaparte's Kaputt

Michèle Coury

\section{(2) OpenEdition} Journals

Édition électronique

URL : http://journals.openedition.org/cei/3378

DOI : $10.4000 /$ cei.3378

ISSN : 2260-779X

Éditeur

UGA Éditions/Université Grenoble Alpes

Édition imprimée

ISBN : 978-2-84310-370-4

ISSN : $1770-9571$

Référence électronique

Michèle Coury, « La beauté et l'enfer se disent à voix basse : dire le mal et témoigner dans Kaputt », Cahiers d'études italiennes [En ligne], 24 | 2017, mis en ligne le 28 février 2017, consulté le 26 mars 2021. URL : http://journals.openedition.org/cei/3378 ; DOI : https://doi.org/10.4000/cei.3378

Ce document a été généré automatiquement le 26 mars 2021.

(c) ELLUG 


\title{
La beauté et l'enfer se disent à voix basse : dire le mal et témoigner dans Kaputt
}

\author{
La bellezza e l'inferno si dicono a voce bassa: dire il male e testimoniare in \\ Kaputt \\ Beauty and the Inferno "in a Low Voice": Voicing Evil and Bearing Witness in \\ Malaparte's Kaputt
}

Michèle Coury

\section{À la mémoire de mon père}

1 Cette réflexion «La beauté et l'enfer se disent à voix basse : dire le mal et témoigner dans Kaputt " part d'un constat et d'une référence : référence à La bellezza e l'inferno, titre d'un recueil d'articles de l'écrivain-reporter très contemporain Roberto Saviano, non sans rapport avec certains écrits de Curzio Malaparte qualifié par son dernier biographe Maurizio Serra de «inviato speciale nella terribilità della Storia ». Voici ce que nous dit Saviano et qui est pertinent par rapport à notre propos :

Il peso specifico della parola letteraria è determinato dalla presenza della scrittura nella carne del mondo o dall'assenza di carne, invece, per alcuni... In qualche modo questa è la differenza tra ciò che è cronaca e ciò che è letteratura. Non l'argomento, neanche lo stile ma questa possibilità di creare parole che non comunicano ma esprimono, in grado di sussurrare o urlare, di mettere sotto pelle al lettore che ciò che sta leggendo lo riguarda... non mi riguardano belle storie incapaci di mettere le mani nel sangue del mio tempo ${ }^{1}$.

Quant au constat fait en tant que lectrice de Malaparte, il est le suivant : à l'intérieur de son œuvre narrative, on peut observer la récurrence d'un certain nombre de figures rhétoriques, plusieurs figures de la répétition surtout (l'épanalepse, l'anaphore, l'anadiplose qui créent un rythme incantatoire), l'usage excessif de l'adverbe «meravigliosamente » qui, joint à n'importe quel adjectif à la connotation péjorative, 
assure à la fois l'oxymore déconcertant et le glissement du réalisme à la vision surréelle. Mais aussi la répétition insistante de certains stylèmes: les personnages, humains, animaux ou même créatures surnaturelles dans les récits de Malaparte souvent se regardent ou regardent fixement, tels ces Anges inquiétants qui envahissent la prison du narrateur durant la nuit, marchent au plafond la tête en bas, en le regardant fixement ${ }^{2}$. Lorsqu'une question de vie ou de mort est en jeu, pourrait-on ajouter, en lien avec ce qui va être développé, les victimes ou ceux qui vont mourir regardent autrui et nous regardent nous lecteurs fixement ${ }^{3}$. Aussi le stylème " guardare fisso » est-il extrêmement récurrent, joint fréquemment à cet autre stylème qui lui dispute le plus grand nombre d'occurrences: "a voce bassa"; les personnages émettent certaines sentences "à voix basse ", nous précise le texte, ce qu'on pourrait appeler au théâtre une didascalie sonore ${ }^{4}$. Cette précision se rapporte au narrateur mais aussi souvent à ses interlocuteurs divers, rencontrés au cours de cette traversée de l'Europe et de la guerre que sont les romans principaux. Ces deux expressions profondément liées, relevant du même champ sémantique, l'une étant en quelque sorte la correspondance visuelle de ce qu'est l'autre sur le plan sonore, ont pour fonction, semble-t-il, de solliciter particulièrement l'attention du lecteur. Dans le cadre de cette étude, je m'attacherai plus précisément à la didascalie sonore et son usage, essentiellement dans le roman Kaputt. Le recours à cette expression est trop fréquent pour qu'il s'agisse d'une simple insistance rhétorique et il nous semble donc opportun de le questionner.

3 Cette étude pourrait prendre en compte l'intégralité de l'œuvre narrative de Malaparte. Si l'on considère déjà les deux œuvres majeures Kaputt-La pelle comme les deux volets d'un même diptyque, on remarquera que cette expression "a voce bassa » ouvre et conclut l'ensemble : «sono i cavalli del Tivoli, il lunapark che è davanti allo Skansen » dit à voix basse le prince Eugène, premier interlocuteur du narrateur, ouvrant le récit dans Kaputt, lors d'une claire journée automnale à Stockholm ${ }^{5}$. On note par ailleurs que la même précision sonore représente les derniers mots de La pelle, accompagnant le paradoxe qui conclut le roman, paradoxe adressé par le narrateur à son ami, soldat américain, qui repart pour les États-Unis :

«L'Europa è un mucchio di spazzatura» disse Jimmy «un povero paese vinto. Vieni con noi. L'America è un paese libero».

Non posso abbandonare i miei morti, Jimmy [...] i miei morti non possono pagarsi il biglietto per l'America, sono troppo poveri. Non sapranno mai che cosa è la ricchezza, la felicità, la libertà. Sono vissuti sempre in schiavitù; hanno sempre sofferto la fame e la paura [...] è il loro destino, Jimmy. Se tu sapessi che Cristo giace fra loro, fra quei poveri morti, lo abbandoneresti?

«Non vorrai darmi a intendere» disse Jimmy «che anche Cristo ha perso la guerra».

- «È una vergogna vincere la guerra» dissi a voce bassa ${ }^{6}$.

Où l'on voit que la sentence-coup de poing provocatrice, transgressive, sur l'ambivalence du vainqueur se dit à voix basse. Il s'avère d'emblée que cette expression si fréquente dans l'œuvre narrative encadre la façon de dire, de raconter et comme nous le verrons, la façon de témoigner. Tout commence et se conclut à voix basse.

Avant d'entrer plus avant dans notre sujet, il convient de rappeler quelques points relatifs à Malaparte dans cette période du deuxième conflit mondial. Le $1^{\mathrm{er}}$ juin 1940, Malaparte a été rappelé sous les drapeaux, il rejoint en uniforme de capitaine le $5^{\mathrm{e}}$ régiment des Alpini et obtient d'être nommé envoyé spécial du Tempo. Autre commanditaire, le Corriere della sera publiera également le récit de la bataille des Alpes ${ }^{7}$. 
Pour Malaparte, qui fut un héros de la première guerre, ce qui se joue surtout concerne la conviction que son rôle est désormais de témoigner. Par la suite, entre 1941 et 1943, en tant que correspondant de guerre, il suit les troupes allemandes à travers l'Europe centrale, à travers la Roumanie, la Pologne, la Yougoslavie, l'Allemagne, jusqu'à l'Ukraine et la Russie avec notamment l'épisode du siège de Leningrad dans le cadre du plan d'invasion germanique de la Russie, pour atteindre la Suède, la Finlande, le Grand Nord, la Laponie. Tant de correspondances de Malaparte ont pour toile de fond ces jours et ces nuits interminablement confondus sous le pâle soleil arctique. L'accomplissement de ce périple est entrecoupé de fréquents retours en Italie.

\section{La structure du récit s'annonce à voix basse}

6 Pour ce qui est de ces premiers mots prononcés à voix basse qui ouvrent le récit de Kaputt, nous remarquerons que la référence aux chevaux faite par le prince Eugène à voir déjà avec le titre de la première partie du roman «I cavalli » et en cela on peut dire que la structure de Kaputt est annoncée dans ce premier murmure qui en laisse deviner le caractère cryptique ${ }^{8}$.

7 Le roman est divisé en 6 parties qui ont toutes pour titres des noms d'animaux : les chevaux, les rats, les chiens, les oiseaux, les rennes, les mouches. Ces parties se divisent elles-mêmes en différents chapitres, trois ou quatre, qui contiennent chacun un rappel implicite ou explicite, littéral ou métaphorique, analogique, symbolique à l'animal-titre de la partie. Chaque partie part d'une situation spatio-temporelle précise où se distingue un personnage central qui va être l'interlocuteur privilégié du narrateurtémoin. Il s'agit de personnages réels, empruntés à la réalité de l'histoire que Malaparte a rencontrés au cours de sa traversée de l'Europe : par exemple Eugène de Suède, le frère du roi Gustave $V$, à Stockholm dans la première partie, le nazi Hans Frank à Cracovie et Varsovie dans la deuxième partie, le comte Augustin de Foxá à Helsinki dans la troisième partie («I cani »). À l'intérieur de chaque cadre narratif propre à chaque partie se déploient un certain nombre de récits-gigognes encastrés les uns dans les autres, le narrateur tout en parlant à son interlocuteur, se souvenant d'une autre situation vécue à un autre moment. À travers cet emboîtement des récits, le narrateur presque toujours présent, presque toujours témoin dans les différentes situations qu'il évoque, se transporte ainsi par le biais du récit, d'un endroit à l'autre, tantôt en compagnie de diplomates, de seigneurs de la guerre dans leurs palais dorés, tantôt dans la dure réalité de la guerre, près de soldats, près d'humbles personnes que la violence a frappées ou va frapper.

8 Arrêtons-nous un instant sur cette structure du récit en noms d'animaux à laquelle nous introduisent à voix basse les mots du prince Eugène, désignant trois chevaux blancs, tristes et beaux, descendant vers la mer : «sono i cavalli...». Le nom d'animaltitre de la partie ouvre souvent sur des récits qui font littéralement référence à l'animal en question : ainsi dans la première partie intitulée «I cavalli » est-il fait référence, dans l'un des passages les plus célèbres du roman, le chapitre intitulé "I cavalli di ghiaccio ", à de vrais chevaux saisis et gelés dans la glace du lac Ladoga, lors d'une bataille entre Russes et Finlandais. L'après-bataille nous livre cette vision hallucinante et dantesque de têtes de chevaux figés émergeant de la glace, sur lesquelles pendant quelque temps viendront s'amuser les soldats comme sur un manège de chevaux de bois. Dans la partie intitulée "I cani », il est entre autres question de chiens bardés 
d'explosifs que l'on dresse à aller se glisser sous les chars ennemis. Inversement dans la partie intitulée «I Topi » il n'est de rats que les juifs, comme cet enfant de Varsovie cherchant à sortir par un trou du mur du ghetto, pour trouver de la nourriture, visé comme un rat par le fusil du gouverneur Hans Frank, en promenade autour du ghetto avec sa suite dont fait partie le narrateur Malaparte ${ }^{9}$. Cette identification rat-enfant juif éclaire a posteriori le titre du chapitre précédent «I topi di Jassy» concernant le pogrom advenu dans cette ville de Roumanie et commis sur ordre des autorités locales, par la police et une partie de la population (900 tués et 5500 déportés à l'est). La partie intitulée « Gli uccelli » joue également sur les comparaisons : les soldats allemands dans les campagnes de Bessarabie vont à la chasse aux jeunes filles juives comme on va à la chasse aux volatiles. Elle joue aussi, tout du long, sur l'analogie inattendue entre les oiseaux et les yeux, à partir de quelques phrases surréalistes prononcées par un des personnages : «Gli occhi sono uccelli prigionieri ${ }^{10}$. » Dans cette partie, il est donc aussi question de soldats aux yeux grand ouverts ${ }^{11}$ car ils n'ont plus de paupières et qui parlent à voix basse, de chats auxquels les jeunes SS arrachent les yeux dans leur apprentissage de la cruauté, de l'officier allemand dont l'œil de verre est le seul qui ait quelque chose d'humain. Avant même d'avoir lu le roman, on suppute que chaque animal-titre d'une partie doit être en quelque sorte l'animal totémique de l'un des personnages. Quoi qu'il en soit, le narrateur n'est jamais à court d'histoires se rapportant de façon littérale ou analogique au nom de l'animal-titre de la partie, cédant parfois à la prière de ses interlocuteurs ou interlocutrices. Ainsi, la demande pressante de Louise (Louise de Prusse, petite fille du Kaiser Guillaume II, venue attendre le narrateur à la station de Postdam) ouvre-t-elle le premier récit de la partie consacrée aux «oiseaux»: " "Mi racconti la storia dell'occhio di vetro" disse all'improvviso Louise a voce bassa ${ }^{12}$. " Cette incitation à raconter nous rappelle que l'enchaînement des récits peut se faire selon cette didascalie sonore. Il nous apparaît qu'il y a bien un lien intime et secret entre la structure du roman, la modalité sonore de ce "à voix basse » et la substance même des récits.

En règle générale, indépendamment de notre sujet et si l'on se réfère à l'acception courante de cette expression "à voix basse ", à quoi correspond-elle? On parle à voix basse pour ne pas être entendu de tous, pour révéler un secret, ou parce que la simple parole relève d'une transgression ou d'un interdit que l'on se pose à soi-même ou que les autres nous imposent, une dangerosité qui est le sceau du mal. On parle à voix basse pour médire, pour mal-dire ou parce que notre propre parole risque de porter «malheur ». Dans tous les cas, on porte littéralement une male-diction. Ainsi le comte de Foxá ministre d'Espagne en Finlande, personnage truculent de cette galerie de portraits, catholique superstitieux, répugne-t-il à prononcer à voix haute certain mots, certaines paroles magiques comme celle désignant un serpent "culebra", qu'on ne peut dire qu'à voix basse parce qu'elle peut entraîner la mort de quelqu'un de l'entourage :

«Per l'amor di Dio tacete!» gridò de Foxá. «Non pronunciate quella parola a voce alta. Ė una parola terribile che va detta a bassa voce, così...» e movendo appena le labbra, mormorò «culebra» [...] «È una parola che porta digrazia. Qualcuno di noi morirà stanotte, o qualcuno non lontano da noi $»^{13}$.

10 La modulation de la voix basse est également une façon d'attirer l'attention de son interlocuteur, une façon de le captiver pour mieux lui faire quelques révélations de la plus haute importance. Avec son sens de l'observation extrêmement pointu, Malaparte qui excelle dans le portrait (dans chacune des parties ressort une personnalité en 
particulier dont Malaparte fait le portrait) traque jusqu'à la moindre nuance, le moindre éclat ou lueur qui passe dans les yeux mais il est également très attentif à l'éclat des voix, au ton de la voix. À la cour de Galeazzo Ciano à Rome, au club de golf, les mondains, les diplomates et les starlettes s'interpellent à voix haute dans un mélange de légèreté, de ragots, de vanité et de vacuité. Le parler à voix basse se situe entre l'éclat des voix et le silence, la "chasteté du silence ${ }^{14}$ » sur lequel nous reviendrons. Pour résumer, dès qu'un personnage parle à voix basse, c'est bien là qu'on doit tendre l'oreille car c'est bien là qu'il y a quelque chose à entendre.

11 Dès la première partie consacrée aux chevaux et dès le premier chapitre, la didascalie sonore «à voix basse " accompagne l'inauguration de thèmes fondamentaux non pas seulement dans Kaputt mais aussi dans l'ensemble de l'œuvre narrative de Malaparte. Ce que le prince Eugène commence par dire à voix basse, c'est sa nostalgie d'un monde et d'un temps disparus, nostalgie cristallisée sur la ville de Paris, le Paris proustien de la Belle Époque : " "Paris était bien jeune alors" disse il principe Eugenio [...] e mi parlava a voce bassa, di quella sua giovane Parigi, di Puvis de Chavannes, dei suoi amici pittori [...] di quei suoi anni felici ${ }^{15}$.» Moment suspendu que celui-ci, avant le déchaînement de la violence au cœur du récit d'un narrateur autre que celui de la Recherche... Dans ce premier dialogue entre le narrateur et le prince Eugène dont les accents semblent proustiens (ne serait-ce que dans le titre du chapitre "Le côté de Guermantes") la référence aux beaux chevaux du parc Tivoli dans la lumière du crépuscule mentionnés à voix basse par le prince ouvre la porte, de façon inattendue, à l'engouffrement de l'horreur dans le récit. De chevaux en cadavres ${ }^{16} \ldots$

A poco a poco qualcosa di amaro nasceva in me. Parole crudeli mi salivano alle labbra che mi sforzavo invano di soffocare. Così, quasi per modo inconscio mi misi a narrare dei prigionieri russi che accecati e abbruttiti dalla fame mangiavano i cadaveri dei loro compagni, sotto gli occhi impassibili degli ufficiali e dei soldati tedeschi. Avevo orrore e vergogna delle mie parole; avrei voluto chiedere scusa al Principe Eugenio di quella mia crudeltà. [...] Una maschera di profonda soffrenza gli copriva il viso. Egli capiva che soffrivo e mi fissava in silenzio con affettuosa pietà. Sentivo che se egli avessse parlato, se mi avesse rivolto una sola parola, se mi avesse toccato la mano, forse mi sarei messo a piangere ${ }^{17}$.

On voit exprimées ici l'horreur et la honte de dire ce qui ne peut être dit sans courir le risque de provoquer un sentiment d'horreur chez celui à qui on le dit. Pris dans une spirale macabre, le narrateur continue de raconter, avec cette surenchère provocatrice dont il est coutumier et qui est l'autre aspect de la honte et de la souffrance, jusqu'à ce que son interlocuteur lui oppose l'injonction au silence: "“Taisez-vous" disse il principe Eugenio a voce bassa. Disse soltanto "Taisez-vous" ${ }^{18}$. "

\section{Le sacrifice animal}

C'est à la fin de cette première partie, à la fin de cette conversation à tiroirs avec le prince Eugène, à la fin de cet enchaînement de récits que le narrateur délivre le sens, l'image onirique visionnaire qui donne une clef à la structure de l'ensemble du récit, celle d'un rêve qu'il fait fréquemment; la crucifixion d'un cheval, le sacrifice d'un Christ-cheval, ce qu'il appelle un « Golgota bestiale ${ }^{19}$ :

Lasciate che vi racconti uno strano sogno. È un sogno che turba spesso le mie notti.

[...] Sulla cima del monte sorge una grande croce. Dalle braccia della croce pende crocifisso un cavallo. I carnefici arrampicati sulle scale danno gli ultimi colpi di 
martello. [...] Il cavallo crocefisso dondola la testa qua e là, e nitrisce dolcemente. La folla piange in silenzio. Il sacrificio del Cristo-cavallo, la tragedia di quel Golgota bestiale: vorrei che mi aiutaste a chiarire il senso di questo sogno. La morte del Cristo-cavallo non potrebbe rappresentare la morte di tutto ciò che v'è di puro e di nobile nell'uomo? Non vi pare che questo sogno si riferisca alla guerra ${ }^{20}$ ?

La référence au sacrifice de l'animal, très présente dans le symbolisme religieux judéochrétien à travers le sacrifice de l'Agneau, parcourt Kaputt et, plus largement, l'ensemble de l'œuvre narrative de Malaparte, de part en part, suggérant dès à présent ce qui sera explicité un peu plus avant dans le récit: la relation sémantique et structurelle dans le récit entre le mot allemand Kaputt de registre familier («cassé », " détruit », " épuisé ») et sa vraisemblable étymologie hébraïque Koppâroth qui renvoie au sacrifice de la victime, ainsi que le rappelle l'auteur lui-même en exergue au roman et dans l'avertissement qui précède le roman lui- même ${ }^{21}$. L'étymologie est encore explicitée dans le corps du récit, au chapitre des oiseaux. À Louise de Prusse, le narrateur demande : «Lei conosce l'origine della parola Kaputt? È una parola che viene dall'ebraico kopparôth che vuol dire vittima ${ }^{22}$. » Nous pouvons ajouter une précision à cette étymologie rapportée par Malaparte : avant de désigner la victime elle-même, Koppâroth est l'acte même du sacrifice d'expiation et la parole Kopares en yiddish désigne les animaux sacrifiés pour l'occasion, en l'occurrence généralement des volailles $^{23}$. Rapportées au contexte de la guerre, les victimes constituent pour la plupart les oubliés dans le champ de ruines de l'histoire : par le biais de la référence animale, l'auteur nous rappelle le côté sanglant de la souffrance de la victime, chair animale, chair tremblante sacrifiée ${ }^{24}$. Le motif de la souffrance animale est récurrent dans l'ensemble de l'œuvre de Malaparte (nous en voyons l'apothéose dans La pelle avec l'exemplaire chien $\mathrm{Febo}^{25}$ ), souffrance animale qui n'est pas séparée de la souffrance humaine mais qui en constitue l'exemple à la fois le plus viscéral et le plus mystérieux. Jusqu'à l'obsession: dans l'avant-dernière partie intitulée "Le renne » et qui a pour théâtre le Grand Nord, la Laponie, que ce soit dans le récit de Kaputt lui-même ou dans les correspondances journalistiques de l'époque, l'auteur revient à plusieurs reprises sur le sacrifice des rennes en des termes très proches de ceux utilisés pour les chevaux. Une fois par an, à l'automne, les lapons se livrent à un abattage rituel, une sorte de pâques des Lapons; le narrateur erre dans un cimetière d'os et de cornes de rennes " una Roncisvalle bestiale », " un Golgota selvatico ${ }^{26}$ ». Dans un texte écrit en français en 1945, retrouvé dans ses archives, Les métamorphoses, Malaparte revient sur cette obsession du meurtre de l'animal comme sorte de mythe génésiaque de sa propre vision du monde: "L'assassin est toujours un homme me disait un soldat allemand et sa victime, toujours un animal. L'homme assassiné redevient un animal traqué, effrayé. C'est pour cela que l'homme assassiné réveille la pitié. Car il n'est pas un homme mais un animal ${ }^{27}$. » Les hommes dans la guerre notamment meurent comme des animaux, comme des chiens, comme des bêtes de somme. Toutes les fois que les hommes sont décrits en tant que victimes, ils s'animalisent mais ne nous trompons pas sur le sens de ce mot: ils s'élèvent spirituellement pour s'apparenter à l'agneau sacrificiel. Rapportons cet autre passage du texte Les Métamorphoses au sujet d'un rêve qui revient fréquemment chez l'auteur et qui développe le thème du sacrifice christique de l'animal ${ }^{28}$ :

Je me suis toujours demandé si le Christ n'était pas venu sur terre et n'était pas mort sur la croix, pour la rédemption de l'animal dans l'homme, plutôt que de l'homme du péché. Je pense que le péché doit être entendu, comme l'apprentissage de l'homme, de la condition humaine. C'est par le péché qu'Adam, ce merveilleux 
animal, est devenu un homme. La vie dans le paradis terrestre, dans l'Eden, était une communion de vie avec les animaux. Dernièrement j'ai parlé longuement avec le père Bianchi, un prêtre très cultivé, très intelligent [...] Il avait sur les animaux, sur Adam, sur le péché, des vues originales et profondes. Il disait qu'Adam avait «trahi » les animaux. Que c'est par le péché que s'était établie cette irréparable rupture entre les animaux et l'homme. Adam chassé de l'Eden, c'est Adam chassé du monde des animaux, de la grande innocente famille des animaux. [...] C'est au fond cela que j'aime le plus dans le christianisme : cette idée que l'homme est un être déchu parce qu'il a trahi les animaux avec lesquels il vivait en parfaite innocence. De cela peut-être vient ce rêve, si fréquent chez moi et dont j'ai parlé souvent dans mes pages d'un Christ à tête de chien, de cheval, de brebis, de ce Christ à tête d'animal cloué sur la croix. J'en parle même dans Kaputt ${ }^{29}$.

15 Animaliser l'homme, humaniser l'animal : c'est pourquoi, plus d'une fois, le narrateur humanise, dans le sens où il l'élève à la condition de victime digne de compassion, le sanglier ou l'oie servis sur plateau lors de dîners raffinés et destinés à être découpés par le couteau implacable du général gouverneur Frank ${ }^{30}$.

16 La première partie qui déjà, avec les récits des chevaux, voit surgir le thème du sacrifice animal, ouvre donc également grand les portes sur le récit des victimes et des bourreaux. Le dialogue entre le narrateur et le prince de Suède prend fin avec la dernière réplique de ce dernier, prononcée à voix basse. Réitérant son sentiment horrifié devant la cruauté des propos du narrateur, le prince cependant rajoute à voix basse : «Ah si je pouvais souffrir comme vous ${ }^{31}$ ! " Il me semble que dans ce regret ou ce souhait on peut percevoir malgré tout chez le prince l'expression d'une reconnaissance du narrateur; le prince reconnaît dans le narrateur le maître du récit chez qui la cruauté est l'autre aspect de la souffrance, au sens étymologique la capacité d'endurer, de supporter, mais aussi de raconter, d'être le témoin lucide au cœur de la violence de son temps et à qui le sens profond du réel apparaît dans la vision d'un rêve.

\section{L'enfer et la beauté}

17 Les bourreaux parlent à voix basse comme dans une conspiration du Mal, avant de mettre à exécution leur projet ténébreux. Ainsi, dans cet épisode se situant dans le kolkhoze d'un village en Ukraine («I cani ») des soldats allemands demandent à des prisonniers russes de faire un exercice de lecture sur un journal. Qui arrive à bien lire, pourra devenir secrétaire dans un bureau du camp, qui lit mal travaillera la terre. À la fin de l'exercice, après un conciliabule à voix basse entre les officiers allemands, il est décidé de faire fusiller tous ceux qui ont bien lu: «Bisogna ripulire la Russia di tutta questa marmaglia letterata. I contadini e gli operai che sanno leggere e scrivere troppo bene, sono pericolosi ${ }^{32}$. »

18 Les victimes elles aussi parlent à voix basse, pour avertir d'une menace effroyable ou entériner l'implacable condamnation qui s'est refermée sur elles sans espoir de salut. Nous mettrons particulièrement en évidence à l'intérieur de ce cortège de l'apocalypse deux catégories de victimes dont le narrateur témoin recueille la parole : les juifs, pour des raisons évidentes afférentes à cette période de l'histoire, et les femmes qui sont, de tout temps, les premières bafouées dans leur dignité et leur intégrité, en temps de guerre.

19 Ainsi, revenons à Jassy en Roumanie. La partie intitulée « I Topi » est particulièrement intéressante de ce point de vue quant à la vertigineuse mise en abîme du rapport 
bourreaux-victimes et quant à la virtuosité du narrateur-témoin. Le cadre à l'intérieur duquel vont se dérouler des récits est constitué des palais de résidence du général gouverneur de Pologne Hans Frank, à Cracovie puis à Varsovie lors de réceptions mondaines.

Au début de l'évocation de tous ces dignitaires nazis flanqués de leurs épouses qui se trouvent au dîner, il nous est précisé que Frank, entre patelin et menaçant, parle à voix basse avec un air de mystère ${ }^{33}$. Ce soir-là se trouve également présent l'homme d'Himmler, le chef de la Gestapo qui précise à voix basse que lui aussi est un ami du peuple polonais ${ }^{34}$. À Varsovie, la conversation porte sur le peuple polonais et finit inévitablement par arriver sur les juifs du ghetto, lesquels, dit-on, jouissent d'une liberté absolue mais se complaisent dans la saleté, dans des conditions de vie déplorables malgré l'effort d'aménagement et d'organisation rationnelle des autorités allemandes. Pour le narrateur-témoin, le tour de force est d'insérer la parole des victimes à l'intérieur du discours des bourreaux dans une mise en abyme où le récit tend aux bourreaux un miroir dans lequel ils refusent de se reconnaitre. Car c'est bien à tous ces dignitaires nazis que le narrateur en levant son verre s'apprête à raconter le pogrom qui a eu lieu quelques mois auparavant dans la ville de Jassy à la frontière entre la Roumanie et la Russie. Le narrateur évoque la tension qui règne alors dans la ville, avant le déferlement de la violence sauvage ${ }^{35}$. Les paroles prononcées à voix basse par Kane, un épicier juif, traduisent le sentiment d'une menace imminente : " "La gente a Jassy è inquieta; tutti si aspettano qualcosa di brutto" dice Kane. Parla a voce bassa, guardando sospettoso verso la porta ${ }^{36}$. » Puis plus tard, dans une scène entre le rêve et la réalité, des silhouettes noires sortent de l'ombre parmi lesquelles celle d'un vieil homme aux yeux fixes et blancs comme ceux d'un aveugle, qui s'adresse au narrateur en ces termes:

Era un vecchio [...]. Mi guardò a lungo in silenzio, poi disse a voce bassa : «Domnule capitan, un terribile pericolo ci pende sul capo. Non avvertite la minaccia che grava su di noi. Le autorità romene stanno preparando un feroce pogrom. Il massacro può aver inizio da un momento all'altro. Perchè non ci aiutate? Che dobbiamo fare?» «Io non posso far nulla» dissi «[...] Che cosa potrei fare? Chi volete che mi dia ascolto?» «Ma fate qualcosa, ma muovetevi dunque!» disse il vecchio a bassa voce, con trattenuta violenza. «Io ho perso l'abitudine di agire» risposi. «Sono un italiano, non sappiamo più agire; non sappiamo più assumere alcuna responsabilità, dopo 20 anni di schiavitù» ${ }^{37}$.

Dans ces paroles prononcées à voix basse, le narrateur reconnaît la musicalité presque féminine d'une voix surgie du passé ; celle du directeur de la prison Regina Coeli où il séjourna quelques années auparavant et tenta de se suicider. À celui qui lui sauva la vie quelques années auparavant, le narrateur ne peut offrir maintenant que son impuissance ${ }^{38}$. Inexorablement, quelques heures plus tard, le massacre a lieu, à coups de crosse, de couteaux, de fusillades, dans l'horreur et le chaos. Une fois terminé le récit du narrateur sur les horreurs de Jassy, Frank prétend distinguer la haute civilisation allemande, de la barbarie des roumains : « La Germania è un paese di civiltà superiore e aborre da certi metodi barbarici [...] noi imitiamo l'arte del chirurgo, non mai quella del macellaio ${ }^{39}$.» Le narrateur reprend son récit... Après le pogrom, il évoque un épisode de la déportation des juifs, par wagons à bestiaux, dans un village de Moldavie, Podul Iloaiei. Une fois arrivé à destination, lorsqu'on ouvre la porte du train, les occupants sont déjà morts suffoqués et s'écroulent sur le consul d'Italie que le narrateur a accompagné sur place. Alors que les détrousseurs de cadavres sont déjà à l'œuvre, un petit groupe de juifs résignés rassemblés autour du rabbin échangent 
quelques paroles avec le consul et le narrateur. L'un d'eux a séjourné en Italie dans sa jeunesse et voudrait tant revoir ce pays. Ses dernières paroles à voix basse, alors que la voiture du narrateur s'éloigne, sont pour rappeler qu'il a appris par cœur la Divine Comédie et il se met à réciter les premiers vers de l'Enfer... dans une autre mise en abyme :

Rimanemmo a lungo seduti, tacendo nella stanza piena di mosche. Poi Sartori si alzò, e tutti uscimmo in silenzio. Mentre salivamo in macchina, l'ebreo col pizzo alla Napoleone III appoggiò la mano sul braccio di Sartori, e disse umilmente, a voce bassa: «E pensare che so a memoria tutta la Divina Commedia» e si mise a declamare: Nel mezzo del cammin di nostra vita...

La macchina si mosse, e il gruppo degli ebrei vestiti di nero scomparve in una nuvola di polvere ${ }^{40}$.

La référence à Dante nous invite à la lecture de l'Enfer dans l'enfer décrit au fil des pages dans Kaputt et étant donné que la plupart des récits ont lieu dans les terres du Nord, dans des déserts de neige et de glace, la référence intertextuelle au neuvième cercle et aux eaux glacées du Cocyte s'impose plus particulièrement : «Io pensavo a quelle antiche icone della Carelia, dove l'Inferno è rappresentato non dalle vive e benefiche fiamme, ma da blocchi di ghiaccio, nei quali i dannati stan confitti ${ }^{41}$. " La citation du premier vers du premier chant de l'Enfer de Dante récité par l'une des victimes juives, la référence explicite au fond gelé de l'enfer dans l'iconographie de la Carélie, zone frontière entre la Russie et la Finlande permettent rétroactivement de faire le lien avec le premier chapitre «I cavalli » dominé par l'évocation du sacrifice animal. Dans ce chapitre a lieu l'évocation d'une bataille entre Russes et Finlandais dans la forêt de Raikkola en Carélie justement, qui entoure le lac Ladoga : les chevaux de l'artillerie soviétique pris de panique, cherchant à fuir les flammes de l'incendie qui les assaille, se précipitent vers le lac où le gel aussi soudain que féroce va les saisir. Scène déjà mentionnée plus haut dans cette étude, seules les têtes des chevaux aux crinières glacées comme coupées net, semblent reposer dans une vision surréelle et infernale sur la plaque de glace du lac gelé, tout comme n'émergent que les têtes des damnés hors des eaux gelées par les ailes de Lucifer, dans les derniers chants de l'Enfer.

Il giorno dopo, quando le prime pattuglie di sissit, dai capelli brucciacchiati, dal viso nero di fumo, camminando cauti sulla cenere ancora calda attraverso il bosco carbonizzato, giunsero sulla riva del lago, un orrendo e meraviglioso spettacolo apparve ai loro occhi. Il lago era come un'immensa lastra di marmo bianco, sulla quale eran posate centinaia e centinaia di teste di cavallo. Parevano recise dal taglio netto di una mannaia. Soltanto le teste emergevano dalla crosta di ghiaccio. Tutte le teste erano rivolte verso la riva. Negli occhi sbarrati bruciava ancora la fiamma bianca del terrore. Presso la sponda, un groviglio di cavalli ferocemente impennati sorgeva fuor dalla prigione di ghiaccio ${ }^{42}$.

Si Malaparte emprunte bien des éléments à la scénographie dantesque, le propos théologique est aux antipodes. Dans l'enfer de Kaputt, les suppliciés ne subissent pas le juste châtiment divin mais la dévastation arbitraire d'une nature indifférente à la cruauté et à la souffrance du vivant. Le déluge de feu et de glace qui ne connaît ni coupables ni innocents emporte dans un même sacrifice humains et animaux, pris de panique et tremblants d'épouvante.

Dans la partie intitulée " Gli uccelli », le narrateur articule le rapport entre le cadre et les récits, autour du féminin. Il se divertit à provoquer ses amies allemandes Ilse et la princesse Louise de Prusse qui est du côté de la race des seigneurs de par sa haute lignée germanique : elle reste néanmoins accessible au sentiment de culpabilité et de 
honte qu'on exprime à voix basse, se distinguant des dignitaires nazis qu'elle côtoie elle aussi dans les salons et qui jouent joliment du piano. Il se dit cette phrase si bien adaptée à bon nombre de personnages évoqués par le narrateur : «Ce que la guerre a de plus horrible est justement ce qu'elle a de plus gentil. Je n'aime pas voir sourire les monstres ${ }^{43}$ ", tel Ante Pavelic le duce croate, à la voix si douce et musicale, un brave homme à la grande délicatesse de sentiments, qui déclare vouloir garantir la bonté et la justice et qui néanmoins conserve dans un panier ce que le narrateur identifie au prime abord comme des huitres et qui s'avère être des yeux humains, arrachés aux ennemis par ses fidèles oustachis ${ }^{44}$. "J'ai honte de moi [...] Nous devrions tous avoir honte de nous ${ }^{45}$ » sont les seules paroles prononçables à voix basse par Louise, née d'une Allemagne monstrueuse mais qui, par l'emploi de ce «nous » pluriel, veut élargir la honte et la responsabilité à l'ensemble de l'Europe dévastée.

C'est par un vers de Shakespeare tiré du sonnet 54 que s'insinue dans le récit le thème de la corruption généralisée, du pourrissement d'une civilisation, d'une commune marche vers la mort des vainqueurs et des vaincus quand l'histoire nous apprendra que les vainqueurs d'aujourd'hui seront les vaincus de demain.

Quelle image mieux que celle de la corruption de la beauté féminine pourrait-elle traduire cette idée ? «"Anche le rose morte" disse Ilse, "sono immortali". "Shakespeare amava il loro morto odore di rosa" dissi "of their sweet deaths are sweet odors made" beauté n'est évoquée que pour annoncer sa propre corruption.

Le narrateur offre à ses amies allemandes Louise et Ilse deux récits de femmes, l'un concernant une femme appartenant (encore) au camp des vainqueurs, l'autre concernant des filles appartenant au camp des vaincus, les renvoyant l'un à l'autre dans une commune histoire de désespérance et de destruction. L'histoire de l'italienne Giuseppina Antinori, mariée à un haut fonctionnaire du Reich, le baron von Stum et l'histoire des jeunes filles juives de Soroca en Bessarabie arrêtées et enfermées dans un bordel, à la disposition des soldats et des officiers allemands. (Malaparte reprendra ce thème de la femme contrainte à se prostituer aux vainqueurs, en inversant le rapport de forces dans la pièce de 1954 Anche le donne hanno perso la guerra.) Dans le cas de Giuseppina von Stum, c'est toute la nostalgie de l'Italie qui interpelle le narrateur dans ce beau visage triste et las, rencontré lors d'un dîner à Berlin à l'ambassade d'Italie. Durant tout ce premier dîner, Giuseppina déconcertante et mystérieuse distille à voix basse quelque mystérieuse prémonition (" "les papillons aiment mourir” disse Giuseppina von Stum a voce bass $a^{47} »$ ), si différente des femmes de diplomates allemands orgueilleuses des souffrances, des misères, des privations de leur peuple ${ }^{48}$. Quand le narrateur la rencontre par hasard quelques mois plus tard, il retrouve une femme défaite, brisée par une solitude absolue, exténuée par son esclavage domestique au nom de la patrie, mais toujours avec la nostalgie de l'Italie au cœur : « Così a poco a poco era discesa in fondo all'oscuro e deserto mondo femminile della Germania in guerra, in quel mondo tetro, pieno di angoscia, senza grazia, senza speranza ${ }^{49}$." Aussi le narrateur n'éprouve-t-il pas vraiment de sentiment de surprise lorsqu'il apprend quelque temps plus tard que Giuseppina von Stum s'est défenestrée peut-être dans un ultime souvenir d'une Italie qui n'existe plus : elle aussi prenant son envol comme un oiseau du désastre (pour faire écho à l'animal-titre de cette partie des récits ["Gli Uccelli »]). Son époux, pour seul éloge funèbre, a accueilli la nouvelle en faisant le salut nazi. prostitution implique encore davantage le narrateur en tant que témoin désemparé et 
dont la voix se brise. À l'une des jeunes filles qui lui demande à voix basse, remplie d'une sourde terreur s'ils finiront par la renvoyer à la maison, le narrateur temporise, cherche des mots auxquels lui-même ne croit plus : «Non avevo più fiducia nelle mie parole. Le mie parole erano false e cattive ${ }^{50}$. " S'il est une vérité qui subsiste, c'est celle de la main de la jeune fille qu'il tient entre les siennes et qui tremble comme un oiseau apeuré : «La sua mano tremava come un uccello spaurito ${ }^{51}$. »

«Arrivederci, Susanna, dissi a voce bassa » et dans cet au revoir murmuré à voix basse, le narrateur-acteur abdique de son éloquence ${ }^{52}$. Le ciel est rempli d'étoiles mais ce sont des étoiles froides qui n'empêchent pas les ténèbres. Tout est dit de ce qu'il adviendra réellement, non pas le retour à la maison mais la fusillade et pire encore, l'oubli, l'insignifiance d'un déchet balayé : « Non servivano più a nulla. Erano ridotte come stracci. E poi, erano ebree ${ }^{53}$ ", commente un officier allemand. À la fin de ses récits, dans le retour au cadre narratif, le narrateur délègue à la princesse Louise de Prusse la formulation de la honte et de la pitié : «"Parfois j'ai honte d'être femme" disse Louise a voce bassa $a^{54}$.»

\section{La prémonition de l'anéantissement des bourreaux et des victimes, des vainqueurs et des vaincus}

Durant l'été 1942, Malaparte se trouve en Laponie à la suite des troupes allemandes sur le chemin du front de Petsamo. (Il écrit pour le Corriere della sera plusieurs articles qui seront publiés en novembre et décembre suivants.) C'est durant ce séjour sur des terres marquées par le supplice d'un été où le jour ne finit jamais et où le soleil est comme un œil qui ne se ferme jamais, que le narrateur évoque, dans l'avant-dernière partie de Kaputt, le monde des rennes ${ }^{55}$. On voit défiler dans cette région de hauts dignitaires de l'armée allemande, tous ivres-morts la moitié du temps quand ils ne sont pas au combat: le général Dietl, conquérant de Narvick, durant la campagne de Norvège, héros de guerre allemand, le général Mensch qui commande l'aviation sur tout le front Nord, le général von Heunert, on entrevoit même Himmler nu dans un sauna comme en train de se dissoudre sous l'eau. Le narrateur comprend que la force terrorisante des Allemands réside dans la rigidité de leur uniforme mais que sous celui-ci se trouve une peau flasque et morte ${ }^{56}$.

31 Le Grand Nord, c'est le territoire des rennes au regard mystérieux, humble et désespéré. On peut même parler d'un "âge du renne ", comme on parle d'un âge préhistorique, une période de l'évolution de la nature où se trouvait du reste le Sud de l'Europe, il y a des milliers d'années ${ }^{57}$. La vie est si rude sur cette terre inhospitalière à cause des conditions climatiques et désertiques que l'homme semble ne pas pouvoir survivre. Le narrateur évoque les bouches édentées, le délabrement du corps, une humanité qui s'animalise et devient sauvage. Dans ce progressif travail de décomposition de l'apparence humaine et qui plus est aryenne, les regards des soldats ressemblent à ceux des rennes. La cruauté allemande semble s'éteindre dans ce regard innocent et triste. Quels sont les bruits qui courent à voix basse sur cette terre extrême? Que les allemands vont perdre la guerre contre les saumons, nobles poissons résistants qui ne se laissent pas prendre:

I salmoni non hanno paura dei tedeschi, li disprezzano. [...] Il salmone è l'animale più nobile del mondo. Sceglierebbe la morte, piuttosto che mancare alle leggi dell'onore. [...] «Vanno in Russia» disse, «e c'è da sperare che non tornino un 
giorno, con la testa rossa». "Siete sicuri che torneranno?» «Torneranno. E più presto di quel che non si pensi» disse Juho Nykanen; e abbassando la voce aggiunse: «Mi potete credere, signore, i tedeschi perderanno la guerra». [...] «Intendo dire la guerra contro i salmoni. [...] Alcuni soldati tedeschi, l'altro giorno, sono stati trovati morti sulla riva del fiume. È probabile che siano stati i salmoni ad ammazzarli, non vi pare ${ }^{58}$ ?»

Plus étonnant encore, le narrateur se souvient de ce qu'il a entendu raconter à voix basse depuis qu'il est en Laponie, quelque chose dont il est même interdit de parler; des soldats allemands éperdus et hébétés en proie à une merveilleuse folie, se donnent la mort.

E mi torna alla memoria quel che ho udito narrare fin da quando sono giunto in Lapponia, quello di cui ognuno parla a voce bassa come di cosa misteriosa, quello di cui è proibito parlare, intorno a quei giovani soldati tedeschi che s'impiccano agli alberi nel folto delle foreste o si sparano nella tempia, o si gettano nelle acque immote dei laghi o si stendono sul tappeto dei licheni, lasciandosi morire nell'astratta, algida, solitudine della foresta ${ }^{59}$.

Même les jeunes seigneurs de la guerre peuvent vouloir mourir, en opposition à ce devoir de l'invincibilité conquérante. Il est intéressant ici de se référer à la substance de certains articles écrits par Malaparte durant ce séjour dans le Grand Nord, notamment sur l'horreur d'une nature ennemie de l'homme, sur le silence sidéral, sur la survivance du renne comme témoignage d'un âge préhistorique, comme le principal protagoniste des mythes héroïques et religieux. Dans un article intitulé "La dignità umana" Malaparte rapporte les propos que lui fait à voix basse le général Dietl qui compare l'aventure du Grand Nord à un absolu vertige, au fait de poser un pied sur l'abîme ${ }^{60}$. Il nous semble que cette solitude sidérale du Grand Nord aux accents métaphysiques contient peut-être un des secrets de ce parler à voix basse, puisque comme nous allons le constater, Malaparte lui-même prend la peine de s'arrêter sur cette expression, à la fois dans un article pour le Corriere della sera intitulé « Addio alle renne » (daté 23 avril 1943) et dans le chapitre de Kaputt, intitulé «Sigfrido e il salmone». Le passage du roman reprend mot pour mot le passage de l'article :

Così mentre parlavamo tra noi a voce bassa - a voce bassa come sempre avviene in quei climi dove la voce umana suona estranea all'uomo, suona falsa, arbitraria, staccata da lui, piena di disperazione, ed è proprio la voce di una angoscia segreta che non trova modo di esprimersi e di esaurirsi che in se stessa, nel suono di sé, nell'eco di sé -, vedemmo trascorrere fra gli alberi, a un centinaio di passi da noi, alcuni animali simili a cani, dal pelo corto, di un grigio color ferro rugginoso. «I lupi» dissero i soldati. Ci passavano vicini, guardandoci con quei loro occhi rossi e lucidi. E pareva che non avessero alcuna paura, alcun sospetto di noi. Nella loro confidenza, non v'era soltanto qualcosa di pacifico ma, direi, qualcosa di distratto, una specie di nobile e triste indifferenza. Correvano senza far rumore, veloci e leggeri, con quel loro passo lungo, agile e morbido. Nulla di ferino era in loro, ma una tal quale nobile timidezza, una specie di orgogliosa e crudelissima mansuetudine. Uno dei soldati alzò il fucile, ma un compagno gli abbassò la canna. V'era in quel gesto una rinunzia, un abbandono di quella crudeltà che è propria dell'uomo. Come se anche l'uomo in quelle disumane solitudini, non trovi altro modo di esprimere la sua umanità che nell'accettazione di una ferinità triste $e$ mansueta $^{61}$.

Voix basse, voix des origines, voix préhistorique de l'homme animal, voix de la solitude primordiale dans l'immensité boréale indifférente à la solitude de l'homme et de l'animal. De façon récurrente dans le roman et dans les correspondances journalistiques, Malaparte insiste sur l'immensité de l'horizon du Grand Nord, sur cette 
lumière incroyablement pure, sur ce désert abstrait où les sens humains n'ont plus de points de repères, sur cette impression d'être suspendu dans le vide. Dans l'œuvre inachevée Mamma marcia, écrite des années après Kaputt l'auteur revient en des termes semblables, à l'obsession des terres du Nord, celles arpentées pendant la guerre, en insistant sur le vide absolu du ciel. Si sous les pieds s'étend l'abîme, comme le disait le général allemand, cet abîme se tient aussi au-dessus des têtes, un vide absolu, un ciel qui a abandonné les hommes, qui est comme le reflet d'un autre univers lointain, totalement étranger, d'une impassible abstraction ${ }^{62}$.

Le guerrier Siegfried des mythes germaniques a pris ce regard de bête en connivence avec la mort. Les vainqueurs seront vaincus, seront détruits, tous détruits et renvoyés à cet abîme de solitude où il n'y a que des vaincus. Le narrateur regarde le général Dietl qui s'est endormi après une beuverie: "Io mi curvo su Dietl, il conquistatore di Narvick, è un eroe della guerra tedesca, un eroe del popolo tedesco. È anch'egli un Sigfrido, è anch'egli Sigfrido e gatto insieme, è un eroe, è anch'egli un koppâroth, una vittima, un kaputt; è una cosa meravigliosa esser morto ${ }^{63}$. " En fait, cette considération vient compléter un passage vu précédemment dans la partie « Gli Uccelli » au moment même où le narrateur donne la clef du titre du roman :

Vi è un momento, ed è un momento che sempre ritorna, in cui anche Sigfrido, l'unico, diventa gatto, diventa koppâroth, vittima, diventa kaputt: è il momento in cui Sigfrido è prossimo alla sua morte, in cui Hagen-Himmler si prepara a cavargli gli occhi, come a un gatto. Il destino del popolo tedesco è di trasformarsi in Koppâroth, in vittima, in Kaputt. Il senso riposto della sua storia è in questa sua metamorfosi da Sigfrido in gatto. [...] deve sapere che tutti siamo Sigfrido, che tutti siamo destinati ad essere un giorno koppâroth, vittime, ad essere kaputt; che per questo siamo cristiani, che per questo anche Sigfrido è cristiano, anche Sigfrido è gatto. Gli imperatori, i figli degli imperatori, i nipoti degli imperatori, debbono anch'essi conoscere certe verità ${ }^{64}$.

La dernière partie du roman qui voit le retour à Rome à la cour de Ciano, puis à Naples après la destitution de Mussolini marque l'invasion des mouches, animal non emblématique de la victime celui-là, mais glorieusement installé sur la putréfaction généralisée. "Finiremo tutti mangiati dalle mosche» dit quelqu'un de l'entourage de Ciano $^{65}$. À Ciano lui-même, le narrateur prophétise une fin inéluctable :

Dovevate tu e gli altri far qualcosa nel 1940 per impedire questa guerra. Far qualcosa, rischiare qualcosa, era quello il momento di vender cara la pelle. Ora la vostra pelle non conta nulla. Ma vi piaceva troppo il potere, questa è la verità. [...] È troppo tardi ormai, affogherete tutti con lui ${ }^{66}$.

En proie à une sorte de délire, le narrateur prophétise en termes ésotériques le déferlement du fléau apocalyptique mais autour de lui, dans le salon mondain, on ne le comprend pas: "Tutti aspettano con terrore il gran Koppâroth, il Kaputt, il gran Gatto $^{67}$ » comme fondant ensemble l'Agneau biblique et la Bête de l'apocalypse. La boucle est bouclée avec l'avertissement au lecteur qui précède le récit lui-même et où Malaparte dégage quelques pistes d'interprétation de son œuvre. Nous y trouvions déjà, à vrai dire, (et la chose se fait plus claire quand on relit cet avertissement après avoir lu le roman), cette fusion d'une notion et de son contraire sous la même appellation :

Kaputt è un libro crudele. La sua crudeltà è la più straordinaria esperienza che io abbia tratto dallo spettacolo dell'Europa in questi anni di guerra. Tuttavia, fra i protagonisti di questo libro, la guerra non è che un personaggio secondario. [...] Il protagonista principale è kaputt, questo mostro allegro e crudele ${ }^{68}$. 

connaît la phrase fameuse dans La pelle que Malaparte met opportunément dans la bouche de son ami américain Jack, dans sa réponse aux militaires français Pierre Lyautey et le général Guillaume, l'un demandant ce qui est vrai dans Kaputt et l'autre déclarant que dans ce roman, Malaparte se moque de ses lecteurs. Ce à quoi Jack répond : «Non ha alcuna importanza se quel che Malaparte racconta è vero o falso. La questione da porsi è un'altra; se quel che egli fa, è arte o no ${ }^{72}$. " 
De plus, l'excès ne trahit pas forcément la réalité de l'histoire : comme le souligne très justement Dominique Fernandez qui s'interroge sur la façon de rendre l'horreur chez Malaparte, l'hyperbole, l'excès lyrique est ce qui est à la hauteur de la démesure des événements : selon la belle paronomase, déformer pour informer ${ }^{73}$. Mais il reste, malgré sa flamboyance, un non-dit dans Kaputt, de l'ordre de l'indicible. Entre l'excès et l'indicible, se tient peut-être le caractère magistral de ce récit ou plus exactement de cet ensemble de récits, qui n'est pas seulement un témoignage sur l'Europe dévastée par la guerre mais aussi un mystère religieux de la nuit des temps, de ceux que l'on dit à voix basse dans une enceinte sacrée. Pour traduire cela, au-delà du reportage et d'une réflexion sur l'histoire, entre le silence et l'insignifiance du bruit, entre mensonge et vérité, Malaparte ne peut emprunter que la voie haute de la littérature. Ce passage du texte critique de Giorgio Bàrberi Squarotti tiré de L'allegoria degli orrori della guerra fermera la boucle avec la citation de Roberto Saviano de notre introduction :

Il personaggio del capitano Malaparte sa che per potere parlare davanti agli inconsapevoli oppure agli indifferenti ancora, oppure ai signori della guerra e della morte, è necessaria la maschera dell'irresponsabile, che tante cose ha visto, innomabili e atroci, ma non può gridare il suo orrore, se vuole che la testimonianza rimanga, sia tramandata alla memoria di chi verrà dopo la guerra a chiedere che cosa è accaduto, e quali sono $\mathrm{i}$ colpevoli, e non potrà credere a quanto atroce è accaduto se non ci sarà il testimone a garantirne la verità; e il testimone, nei tempi attuali non può che essere lo scrittore ${ }^{74}$.

Mais de quel langage secret l'écrivain se fait-il le vecteur à voix basse à travers son témoignage et la création littéraire? Une fois encore, le texte Les Métamorphoses, qui consiste pour une bonne partie en une méditation sur les animaux et sur leurs rapports aux hommes, peut nous éclairer et resserrer le sens autour de la division du roman en noms d'animaux. Dans le passage qui suit, Malaparte mentionne un médecin de sa connaissance à Helsinki :

Le fameux Doctor Feltin, d'Helsinki, en Finlande, a une étrange théorie sur les animaux. Selon lui, les animaux parlaient, jadis, au temps des fables. Peu à peu, dans leur évolution, ils ont renoncé à la parole, comme à une chose inutile. Leurs cordes vocales témoignent de leur langage d'autrefois. Les fables des animaux parlants témoignent aussi de leur ancien langage. L'évolution des animaux est achevée, dit Faltin. Les hommes, on ne sait pas encore où ils vont. Renonceront-ils aussi peut-être au langage ${ }^{75}$ ?

Et, un peu plus loin :

La mort, la mort véritable, celle que les animaux comprennent, ce n'est pas la mort du corps, ni celle de l'esprit. Elle n'a rien à faire, ni avec le corps, ni avec l'esprit. La mort, c'est Dieu. Le monde avec lequel nous sommes en contact, c'est le royaume de la mort, c'est l'enfer, le royaume souterrain, le pays des rêves, des présences, des intuitions, des clameurs nocturnes. Nous n'entendons pas, comme les animaux, ces voix souterraines, le bruit sourd du fleuve Acheronte qui coule sous nos pieds, audessous de notre univers visible, sensible, rationnel. Nous n'entendons pas, comme les animaux, les voix du pays infernal, de notre véritable patrie [...] les animaux sont les intermédiaires entre Dieu et nous. Entre le surnaturel et nous, entre les secrets de la nature et nous. Les animaux nous avertissent de l'approche de la maladie, du malheur, de la mort. Ce sont nos sibylles, nos oracles. [...]

Quelle serait la littérature des chiens, des animaux, si les animaux pouvaient avoir une littérature? J'en ai discuté des heures durant avec Faltin à Helsinki. On serait porté à croire que la littérature des animaux serait une littérature romantique qui aurait pour objet la nature. Une poésie virgilienne, géorgique. Je crois plutôt que la littérature des animaux serait une littérature morale, elle aurait pour objet le monde infernal, la destinée de l'animal et du monde, de l'univers, de la nature. La 
guerre (la chasse), l'aventure en tant que découverte du monde, des forêts, des rivières, des montagnes seraient les arguments de leur épopée. Mais la mort, voilà le grand objet constant de la méditation animale ${ }^{76}$. davantage que les figurants réels et symboliques d'un ensemble de récits mais les tenants d'un langage perdu. Si Kaputt, malgré sa véracité historique en bien des points peut être lu comme un ensemble de fables, c'est aussi parce que le «temps des fables ${ }^{77}$ » est celui où les animaux parlaient.

si c'était eux, les animaux, les véritables narrateurs des récits de Kaputt? Les narrateurs d'un langage perdu que l'écrivain hanté retrouve par fragments apocryphes prononcés à voix basse, entre la raison de l'homme et le mystérieux silence des animaux en prise sur les rumeurs de l'enfer. De quoi parleraient les animaux s'ils parlaient encore? Ils parleraient des couteaux qui s'aiguisent dans les cuisines des palais, des explosions dans le ciel, des déserts de neige à l'aurore boréale, de leur infinie solitude, ils parleraient toujours et encore de ce dont ils ont connaissance depuis la nuit des temps de l'Homme: l'immolation, les persécuteurs et leurs cortèges «d'idoles sanglantes ${ }^{78} »$.

\section{NOTES}

1. R. Saviano, La bellezza e l'inferno, Milan, Mondadori, 2009, p. 240-242.

2. Cette fixité du regard des anges est évoquée au cours du dialogue entre sa mère à l'agonie et le narrateur, lorsqu'il évoque la misère et la solitude des prisons : " "Le carceri sono piene di Angeli, la notte". [...] "Come erano?" disse mia madre. "Erano belli?" "Sì" dissi "erano bellissimi. Avevano occhi lucidi e fissi. Si muovevano lentamente, guardandomi fisso. Ogni tanto si fermavano, mi guardavano fisso. Alcuni si arrampicavano sui muri della cella, camminavano sul soffitto con la testa in giù, guardandomi fisso. Poi si lasciavano cadere a capofitto sul letto. Le mie notti erano una continua lotta di Giacobbe con l'Angelo, la mattina mi risvegliavo sciancato, come Giacobbe".» (C. Malaparte, Mamma marcia, Florence, Vallecchi, 1959, p.95. C'est nous qui soulignons.)

3. Au chevet de sa mère mourante, le narrateur commence à évoquer à voix basse le souvenir du soldat Nazzareno Jacoboni touché par l'éclat d'une grenade lors d'une bataille en Champagne : il sait qu'il va mourir et souffre comme une bête blessée : "Soffriva come una bestia ferita, in silenzio, guardandoci fissi [...] ci guardava uno per uno come fa il cane morente che guarda il padrone, sperandone aiuto. Aveva proprio gli occhi di un cane morente, e nel viso quel sorriso, quell'espressione di bambino che hanno i cani malati, i cani in agonia.» (Ibid., p. 123)

4. Il n'est d'ailleurs pas rare que les deux expressions soient jointes dans le même passage, et ce, dès les premiers récits de Malaparte. Voyons par exemple ce passage du récit Primo sangue où le narrateur se revoit enfant confronté au mystère du sang, son propre sang d'abord, le sang des animaux, le sang qui parcourt les veines de tous les êtres vivants. Durant l'une de ses errances dans la campagne, il croise un garçon qui traîne en laisse un chien galeux, sans doute pour aller le noyer : "Dal modo come mi fissava, capii che portava la povera bestia ad affogare... "Lascialo andare" gli dissi a un tratto a voce bassa. Il ragazzo mi guardava fisso, pareva sordo, non udì, non 
rispose, non si mosse... Poi adagio si curvò, sciolse il laccio, se ne andò in silenzio. » (C. Malaparte, Sangue, Florence, Vallecchi, 1954 (1935), p. 32)

5. C. Malaparte, Kaputt, « Le côté de Guermantes », Milan, Mondadori, 1979 (1944), p. 9.

6. C. Malaparte, La pelle, Milan, Mondadori, 1978 (1944), p.325. Dorénavant, c'est nous qui soulignons systématiquement cette expression dans les citations.

7. M. Serra, Malaparte, vies et légendes, Paris, Grasset, 2011, p. 299-300.

8. Citons cette remarque extraite de la préface à la biographie politique de Malaparte qui met d'emblée en évidence cet aspect de l'œuvre de Malaparte : «Curzio Malaparte fu, in certa misura, uno scrittore criptico, nel senso che le sue pagine, sempre smaglianti, sono da leggere in controluce, hanno significati celati che bisogna scoprire come in una caccia al tesoro.» (F. Perfetti, prefazione a G. Pardini, Curzio Malaparte biografia politica, Milan, Luni, 1998, p. 8)

9. C. Malaparte, Kaputt, « Cricket in Polonia », ouvr. cité, p. 172.

10. Ibid., « Un paniere di ostriche », p. 265.

11. Ces soldats aux paupières nécrosées, brûlées par le froid, illustrent d'ailleurs littéralement le fait de "regarder fixement » dont nous avons déjà mis en évidence les nombreuses occurrences dans l'œuvre romanesque de Malaparte: «Pensavo che quegli infelici dormivano con gli occhi spalancati nel buio, che la loro palpebra era la notte; che attraversavano il giorno camminando con gli occhi sbarrati e fissi incontro alla notte [...] che il loro destino era la pazzia; che soltanto la pazzia avrebbe dato un po' d'ombra ai loro occhi senza palpebre. » (Ibid., "L'occhio di vetro ", p. 254)

12. Ibid., p. 259.

13. Ibid., « La notte d'inverno», p. 186.

14. Ibid., « Le côté de Guermantes », p. 29 (en français dans le texte).

15. Ibid., p. 28.

16. De chevaux en cadavres et aussi en cadavres de chevaux. Dans ses écrits en tant que correspondant de guerre pendant la campagne de Russie, Malaparte insiste sur la vision de carcasses de chevaux, victimes encore plus horrifiantes que les cadavres humains, sacrifiées à la famine des soldats russes: «Il campo di battaglia appare in un disordine indescrivibile [...] I cadaveri russi giacciono a mucchi davanti ai bunker finnici. Ma i resti più raccapriccianti sono le carogne dei cavalli, tagliate a pezzi dai soldati russi famelici. » (C. Malaparte, Corriere della sera, 24 avril 1942, dans E. Ronchi Suckert (éd.), Malaparte, vol. VI, Città di Castello, Ponte alle grazie, 1991, p. 107)

17. Kaputt, « Le côté de Guermantes », p. 17.

18. Ibid., p. 19.

19. Cette vision sacrificielle du cheval est omniprésente dans Kaputt. Nous pensons qu'elle renvoie au thème du sacrifice du cheval attesté dans la pratique rituelle de nombreux peuples, souvent dans le cadre de rites de fertilité : la viande consommée après le sacrifice était censée transmettre à celui qui la mangeait, la force du cheval. Les Vikings notamment effectuaient des rituels de sacrifices de chevaux blancs (blancs comme les beaux chevaux tristes remarqués par le prince Eugène au début du récit) avant d'en consommer la viande (cf. P. Rhys Mountford, Nordic Runes: Understanding, Casting and Interpreting the Ancient Viking Oracle, Rochester, Bear and Company, 2003). S'interrogeant sur l'anthropologie qui, dans ses recherches sur le sacrifice, a toujours escamoté, selon lui, la donnée la plus évidemment pertinente, c'est-à-dire la violence, René Girard rappelle que l'Ashvanedha (littéralement «sacrifice d'un cheval » en sanskrit), important rituel de sacrifice équin dirigé par un roi, dans la religion védique, comporte, en principe, entre autres victimes, l'immolation d'un être humain. «Ce sacrifice humain, toutefois, est mentionné incidemment, comme si de rien n'était. » (R. Girard, Le sacrifice, Paris, Bibliothèque nationale de France, 2003, p. 14.) Pour ce qui est du sacrifice de l'animal lié à la représentation christique, on peut se demander si Malaparte n'a pas été en quelque mesure influencé au moins visuellement par l'exemple le plus troublant de représentation d'animal crucifié (représentations 
plutôt rares, il est vrai) et qui plus est appartenant à l'histoire de Rome : le Christ à tête d'âne ou de cheval découvert en 1836 dans les ruines du palais des Césars au Palatin, gravé au troisième siècle sur un des murs du paedagogium, partie du palais destinée à l'instruction des enfants esclaves, et passé à la postérité sous le nom du " graffite d'Alexamène » (graffito di Alessameno). Il représente un être hybride, un homme à tête d'âne ou de cheval suspendu à une croix. À droite, au-dessus de la croix, on distingue le signe "y ». À gauche, un personnage lui envoie le baiser rituel d'adoration : il se tient au pied de la croix avec, au-dessous de lui, l'inscription en grec «Alexamène adore son dieu ». Ce qui rend ce graffiti antique si fascinant c'est, bien sûr, l'aura de mystère persistant qui l'enveloppe, malgré les gloses diverses sur le sujet. S'agit-il d'une représentation blasphématoire du Crucifié s'adressant à un des adeptes de la nouvelle religion (une autre inscription en latin sur le mur « Alexamenos fidelis » nous fait comprendre qu'il s'agit d'un chrétien) et tournant en dérision la prétendue onolâtrie que les païens attribuaient à la fois aux juifs et aux chrétiens? Ou bien cette représentation n'est-elle en rien une caricature du Christ mais plutôt, de façon positive et affirmée, le fragment hermétique d'un culte gnostique voué à Typhon-Seth souvent représenté avec une tête d'âne et ici superposé sur la figure du Crucifié ? (Voir S. Reinach, Le culte de l'âne, Paris, éd. Ernest Leroux, tome 1: "Cultes, mythes, religions ", 1905, p. 342-346.) Les deux interprétations, quoi qu'il en soit ne sont pas étrangères à notre propos puisque, bien que différentes, chacune rend compte de la charge à la fois occulte et subversive de l'image de l'homme-animal crucifié, aux yeux des païens des premiers siècles. L'évocation de Malaparte semble vouloir réactualiser cet aspect extrêmement dérangeant et scandaleux... pour nos mentalités contemporaines.

20. Kaputt, ibid., «I cavalli di ghiaccio», p.57-58. Précisons en outre qu'à un moment donné, durant sa conversation inaugurale avec le prince Eugène, Malaparte mentionne sa visite à Uppsala, la veille, et le fait qu'il s'est rendu près des tombes des antiques rois de Suède. Une question semble le tarauder, savoir s'il est vrai que dans les temps anciens, les Suédois sacrifiaient les chevaux sur les tombes des rois : « Io pensavo alle teste dei cavalli appese ai rami delle querce di Uppsala, sulle tombe dei re, ai grandi occhi equini colmi di quello stesso umido splendore che hanno gli occhi delle donne quando il piacere o la pietà li illumina. » (Ibid., «Le côté de Guermantes ", p. 20.) Le journal de bord tenu durant le début de l'automne 1942 témoigne de la même conversation et préoccupation : «Ho chiesto al Principe se fosse vero che, in antico, gli svedesi ancora pagani, sacrificassero in quel giorno dei cavalli ai loro dei. Pare che li impiccassero agli alberi.»(C. Malaparte, Giornale segreto, 30 septembre 1942, dans E. Ronchi Suckert (éd.), Malaparte, vol. VI, ouvr. cité, p. 284.) Il y fait à nouveau référence de façon pour le moins imprécise dans une de ses correspondances du front Nord en 1943, ce qui accrédite l'idée que c'est l'image des chevaux par elle-même qui le hante, plus que la référence historique et le souci de son exactitude: "Kurt Franz [...] narra che gli antichi tedeschi impiccavano i cavalli intorno alle tombe dei loro re. Avete visto a Uppsala le tombe dei primi re svedesi? » («Viaggio in Lapponia ", Corriere della sera, avril 1943, dans ibid., p. 345.) Fait-il allusion au site de Gamla Uppsala où avaient lieu des sacrifices rituels avant la christianisation et où le roi officiait en tant que prêtre?

21. «KAPUTT (von hebraischen Koppâroth. Opfer, oder französisch Capot, matsch) Zugrunde gerichtet, entzwei. (Meyer, Conversationlexicon, 1860)». "Nessuna parola, meglio della dura, e quasi misteriosa parola tedesca Kaputt, che letteralmente significa "rotto, finito, andato in pezzi, in malora" potrebbe dare il senso di ciò che noi siamo, di ciò che ormai è l'Europa: un mucchio di rottami. » (C. Malaparte, Kaputt, p. 2, 4)

22. Ibid., "L'occhio di vetro", p. 258. Rappelons que le premier sens étymologique de la parole latine « victima » désigne l'être vivant sacrifié à la divinité.

23. Kopparôth ou kapparot: dans la tradition juive, coutume observée à la veille de Yom Kippour, d'égorger une volaille après l'avoir passée trois fois au-dessus de sa tête, en disant: "Ceci est mon expiation, ceci est mon rachat, ceci est ma substitution. » Avant la cérémonie de transfert 
symbolique de la culpabilité d'une personne à un animal qui était ensuite tué (puis vendu pour une œuvre charitable) on récitait traditionnellement quelques versets du psaume 107 (v. 10-21) où l'on rend grâce à Dieu : le Très-Haut répond à ceux qui l'appellent du fond de leur détresse, même si cette détresse est due à leur dévoiement, à leurs fautes et leur rébellion contre sa loi : «Et il criaient vers Yahvé dans la détresse / de leur angoisse il les a délivrés / il les tira de l'ombre et la ténèbre / et il rompit leurs chaines. / Qu'ils rendent grâce à Yahvé de son amour / de ses merveilles pour les fils d'Adam ! / Insensés sur les chemins du péché, / misérables à cause de leurs fautes, / tout aliment les dégoûtait, / ils touchaient aux portes de la mort / Il envoya sa parole, Il les guérit / à la fosse Il arracha leur vie. » On récitait également des versets du livre de Job (chap. 33) où il est dit que Dieu peut racheter celui qui se consume dans le tourment. Celui qui était déjà près de la fosse reprend vie grâce à la compassion de Dieu. "Quand son âme approche de la fosse / et sa vie, du séjour des morts, / s'il se trouve près de lui un Ange, un Médiateur pris entre mille, / qui rappelle à l'homme son devoir / alors Dieu prend pitié de lui et déclare: / "Exempte-le de descendre dans la fosse / j'ai trouvé une rançon." / Voilà tout ce que fait Dieu, / deux fois, trois fois pour l'homme, / afin d'arracher son âme à la fosse / et de faire briller sur lui la lumière des vivants. » (La Bible de Jérusalem, traduite en français sous la direction de l'École biblique de Jérusalem, Paris, Les éditions du Cerf, 1998.)

24. Rappelons à cette occasion l'amour de Malaparte pour les animaux, tel que l'évoque Maurizio Serra dans sa biographie : «Depuis son enfance, il aime les animaux, les chiens en particulier. [...] la solitude a accentué ce côté de sa nature. Malaparte, nous l'avons vu, est incapable de rapports profonds avec les hommes, et surtout les femmes; la loyauté, sans réserve mais sans esprit critique, d'un animal est le meilleur succédané qu'il puisse trouver à une relation humaine effective, dont il demeure incapable [...] Son amour des animaux est sans doute le seul élément de sa nature où il se mette à nu, parce qu'il ne risque pas d'être dévoilé. » (M. Serra, Malaparte, vies et légendes, ouvr. cité, p. 234.)

25. Le chapitre central du roman La pelle intitulé « Il vento nero » est construit en trois épisodes comme un triptyque, deux épisodes, « latéraux » de part et d'autre d'une figure centrale, le chien bien aimé Febo. Un beau jour, à Pise, au début de la guerre, Febo disparaît et le narrateur, après bien des recherches, finit par le retrouver dans une clinique vétérinaire, éventré, soumis à une expérimentation scientifique : «Era disteso sul dorso, il ventre aperto, una sonda immersa nel fegato. Mi guardava fisso, e gli occhi aveva pieni di lacrime. [...] mi guardava fisso e un dolore atroce mi scavava il petto. "Febo" dissi a voce bassa. E Febo mi guardava con una meravigliosa dolcezza negli occhi. Io vidi Cristo in lui, vidi Cristo in lui crocifisso, vidi Cristo che mi guardava con gli occhi pieni di una dolcezza meravigliosa. » (C. Malaparte, La pelle, ouvr. cité, p. 161.) Au sujet de la structure en triptyque de ce chapitre et le rapport avec le peintre Francis Bacon, nous nous permettons de renvoyer à M. Coury, "Crucifixions en triptyques », Mélanges offerts à Mario Fusco. Chroniques italiennes, $\mathrm{n}^{\circ}$ 3/4, 1996.

26. C. Malaparte, Kaputt, « Sigfrido e il salmone », p. 345-346.

27. C. Malaparte, Les métamorphoses, dans E. Ronchi Suckert (éd.), Malaparte, vol. VI., 1945, ouvr. cité, p. 733.

28. Voir à ce propos, dans la présente revue, l'analyse du film de Malaparte Cristo proibito et l'insistance de certains plans sur des animaux de boucherie, carcasses, tête de veau, boucher qui aiguise son couteau.

29. C. Malaparte, Les métamorphoses, dans E. Ronchi Suckert (éd.), Malaparte, vol. VI, 1945, ouvr. cité, p. 736.

Dans son essai Le bestiaire $d u$ Christ, Louis Charbonnay-Lassay rappelle que longtemps après que Constantin a reconnu le christianisme, l'église reste réfractaire au fait de représenter le corps adoré de son dieu crucifié sur un instrument de supplice infâmant et que l'agneau opportunément remplit cette fonction de substituer le corps du Christ dans cette représentation. «Ce fut l'agneau, son emblème de choix, qui prit sa place au centre de l'arbre empourpré du sang 
rédempteur. Ce fut là, si l'on peut dire, le premier crucifix, le précrucifix des chrétiens. J'en cite ici comme exemple une croix du Musée national de Ravenne qui est du v siècle, et l'Agneau du ciborium de Saint-Marc de Venise, du vi ${ }^{\mathrm{e}}$ siècle, qui est placé entre les deux larrons crucifiés. » (L. Charbonnay-Lassay, Le bestiaire du Christ, Paris, Albin Michel, 2006 (1941), p. 159-160.) L'étude de l'historien rappelle que la coutume iconographique de placer l'agneau sur la croix persiste en Orient comme en Occident, longtemps après que l'Église a accepté la représentation de l'image du corps de Jésus sur l'instrument de son supplice. Différents conciles entre le vil et le $\mathrm{x}^{\mathrm{e}}$ siècle décrètent qu'il y a lieu d'adopter la figure humaine pour la réalisation des crucifix, l'agneau sur la Croix étant insuffisant à exprimer le mystère de la rédemption.

30. « Fuor delle fauci porcine spuntavano le due bianche zanne ricurve: sul dorso lucente e rorido di grasso, dalla cote croccante, screpolata dall'ardore del fuoco vivo, si drizzavano le dure setole nere. Ed io sentii nascermi nel cuore un'oscura simpatia per quel nobile cinghiale polacco [...] alzato il braccio [Frank] affondò un lungo coltello nel dorso del cinghiale. [...] Ma a questo punto, la porta si aprì dolcemente, e sopra un vassoio d'argento entrò l'oca arrosta, rovesciata sul dorso in mezzo a una ghirlanda di patate rosolate nel grasso. [...] Non so perchè, pensai che non fosse stata sgozzata con un coltello, nel buon modo antico, ma fosse stata fucilata contro un muro da un plotone di SS. [...] Oh sì, mi sentivo dalla parte dell'oca, non dalla parte di coloro che gridavano "feuer!" nè di tutti coloro che dicevano: "Gans kaputt! L'oca è morta". » (C. Malaparte, Kaputt, « God shave the King », p. 68-72)

31. Ibid., p. 58.

32. Ibid., « I cani rossi », p. 209.

33. " "Sono un cattolico" disse Frank "un buon cattolico, ma credete che basti? [...] Non avete un'idea di quanto sia difficile governare un popolo cattolico". "Non mi sono mai provato" dissi sorridendo. "Guardatevene bene!" aggiunse Frank curvandosi sulla tavola e parlando a voce bassa con aria di mistero. » (Ibid., « God shave the King », p. 62)

34. Ibid., p. 77.

35. Pour les faits réels advenus à Jassy, voir M. Serra, Malaparte vies et légendes, ouvr. cité, p. 321 ; E. R. Laforgia, Malaparte scrittore di guerra, Florence, Vallecchi, 2011, p. 77. Voir également le témoignage de Lino Pellegrini envoyé par Il popolo d'Italia qui se trouve avec Malaparte durant une partie de ses déplacements, notamment vers le front ukrainien : L. Pellegrini, «Le guerre di Malaparte ", Storia illustrata, $\mathrm{n}^{\circ}$ 192, novembre 1973, p. 56.

36. C. Malaparte, Kaputt, «I topi di Jassy », p. 109.

37. Ibid., p. 118.

38. Ibid., p. 120-121.

39. Ibid., p. 139.

40. Ibid., « Cricket in Polonia », p. 167.

41. Ibid., « La notte d'inverno », p. 201.

42. Ibid., "I cavalli di ghiaccio", p. 53. Cette évocation hallucinée annonce la description du bombardement de Hambourg dans La pelle qui est le pendant du texte des chevaux du lac Ladoga : elle traduit la même obsession du chant dantesque, le même effet de multitude des corps réduits à l'état de têtes émergées et l'on y retrouve certains termes déjà utilisés pour les chevaux dans Kaputt. La scène décrit l'atrocité des effets des bombardements au phosphore effectués par les armées alliées, sur la population civile allemande. La référence à l'Enfer de Dante et à «Dite, la città infernale» est ici explicite: "Anche i tedeschi sono mortali. Ma migliaia e migliaia d'infelici, grondanti di fosforo ardente, sperando di spegnere il fuoco che li divorava, s'eran gettati nei canali che attraversavano Amburgo in ogni senso, e nel fiume, nel porto, negli stagni, perfino nelle vasche dei giardini pubblici, o s'eran fatti ricoprir di terra nelle trincee scavate, per immediato rifugio in caso d'improvviso bombardamento [...] Poichè il fosforo è tale che si appiccica alla pelle come una viscida lebbra, e brucia solo al contatto dell'aria. Non appena quei disgraziati sporgevano un braccio fuor della terra o dell'acqua, il braccio si accendeva come una 
torcia. Per ripararsi dal flagello, quegli sciagurati erano costretti a rimanere immersi nell'acqua o sepolti nella terra come dannati nell'Inferno di Dante. [...] Per alcuni giorni, Amburgo offrì l'aspetto di Dite, la città infernale. Qua e là nelle piazze, nei canali, nell'Elba, migliaia e migliaia di teste, che parevano mozze dalla mannaia, livide dallo spavento e dal dolore, muovevan gli occhi, aprivan la bocca, parlavano. [...] Non appena scendeva la notte, nasceva intorno un bisbiglio, un sussurro, come di vento nell'erba, e quelle migliaia e migliaia di teste guatavano il cielo con occhi accesi di terrore. " (C. Malaparte, La pelle, ouvr. cité, p. 99-100.) Il nous semble intéressant de mentionner un autre texte de Malaparte publié dans Corriere della sera qui correspond de façon inversée à la scène violente des chevaux pris dans la glace et traduit à nouveau une référence implicite au neuvième cercle et plus précisément au chant XXXII dont nous jugeons pertinent de rappeler les vers suivants : "Come noi fummo giù nel pozzo scuro / sotto i piè del gigante assai più bassi, / e io mirava ancora a l'alto muro, / dicere udi'mi: "Guarda come passi; / va sì, che tu non calchi con le piante / le teste de' fratei miseri lassi". / Per ch'io mi volsi, e vidimi davante / e sotto i piedi un lago che per gelo / avea di vetro e non d'acqua sembiante. » (v. 16-24) Cette foisci, le correspondant de guerre Malaparte se met directement en scène : il est intéressant de comparer son attitude à celle du poète florentin qui, faisant fi de l'invitation à passer délicatement au milieu des têtes des damnés, finit par trébucher sur l'une d'elles et, après un échange virulent, armé de son bon droit de se conformer à la justice divine, la saisit violemment par les cheveux pour lui faire dire son nom, le traitre Bocca degli Abati. Le récit de Malaparte est un texte de 1942, écrit pendant la campagne de Russie, en lien avec l'évocation du lac Ladoga dans Kaputt. Malaparte a pris l'habitude d'attendre l'aube sur la rive du lac Ladoga, sur la petite plage d'où partent les patrouilles de skieurs pour les explorations nocturnes. Il observe la plaque de glace qui recouvre le lac peu profond : à certains endroits, la croûte de glace est si épaisse qu'elle touche le fond du lac, des familles entières de poissons se retrouvent piégées dans ces prisons de cristal. Aux premiers signes du dégel, le lac révèle certains de ses secrets : on retrouve les cadavres de soldats finnois morts durant les assauts. Un beau jour, Malaparte qui marche sur la glace du lac comme sur une plaque de verre, au bord du vertige, comme suspendu dans le vide, voit apparaître sous ses pieds des visages fantomatiques pris dans la glace, semblables à des icônes byzantines, des "masques de verre ", non pas les visages des cadavres eux-mêmes, mais leurs traces ineffables et subtiles imprimées dans la glace, un souvenir de ces combattants sacrifiés, sur le point de se dissoudre. Malaparte saisi par ce moment de grâce fugitive, agenouillé sur le sol gelé et transparent, caresse doucement de la main ces «impressions » diaphanes, ce souvenir labile d'êtres disparus et qui vont l'être à jamais, effacés par la lumière du soleil : «Impressi nel ghiaccio, stampati nel trasparente cristallo, apparivano sotto la suola delle mie scarpe una fila di volti umani, bellissimi. Una fila di maschere di vetro [...] Quel che m'appariva nella lastra di ghiaccio era un'immagine meravigliosa, piena di una dolce e commovente pietà. Era come l'ombra delicata e viva, di uomini scomparsi nel mistero del lago. La guerra e la morte hanno talvolta di queste delicatezze misteriose, piene di un alto fiato lirico. La guerra certe volte ha cura di trasformare in bellezza le sue immagini più realistiche, quasi che a un certo punto sia sopraffatta essa stessa dalla pietà che l'uomo deve al suo simile, che la natura deve all'uomo [...] Ero curvo sul ghiaccio. M'inginocchiai, passai con dolcezza la mano su quei volti diafani. » (C. Malaparte, "Autocarri sopra un ponte di ghiaccio ", Corriere della sera, 14 mai 1942, dans E. Ronchi Suckert (éd.), Malaparte, vol. VI, ouvr. cité, p. 128.)

À l'inverse du texte dantesque où le cœur du Visiteur se durcit à proximité des traîtres prisonniers de la glace et de Lucifer et où les rimes se font âpres et rauques, on voit que Malaparte insiste sur cette délicate « pietas » dont il est empreint envers son prochain, envers de jeunes soldats sacrifiés à la guerre. L'enfer de la guerre n'est pas le lieu du contrappasso, n'est pas le lieu de la justice divine mais celui du chaos où meurent aussi et surtout des innocents. Toutefois, de cet enfer même, peut naître la beauté telle qu'elle s'impose au narrateur en cet 
instant d'épiphanie où, dans la révérence envers les morts, semble entamé le mur d'indifférence entre les hommes, entre les hommes et la nature si impénétrable.

43. Ibid., «L'occhio di vetro ", p. 255.

44. " "Sono ostriche della Dalmazia?" domandai al Poglawnik. / Ante Pavelic sollevò il coperchio del paniere e mostrando quei frutti di mare, quella massa viscida e gelatinosa di ostriche, disse sorridendo, con quel suo sorriso buono e stanco: "È un regalo dei miei fedeli ustascia: sono venti chili di occhi umani”. » (Ibid., « Un paniere di ostriche », p. 274)

45. Ibid., " Of their sweet deaths ", p. 275.

46. Ibid., p. 278.

47. Ibid., p. 291.

48. "Le mogli tedesche dei diplomatici tedeschi sono orgoglise delle sofferenze, delle miserie, degli stenti del popolo tedesco. Le mogli tedesche dei diplomatici tedeschi non si buttano dalla finestra, non si ammazzano. » (Ibid., « Le ragazze di Sorocca », p. 296)

49. Ibid., " Of their sweet deaths », p. 294.

50. Ibid., « Le ragazze di Soroca », p. 306.

51. Ibid., p. 306. C'est nous qui soulignons.

52. Ibid., p. 306.

53. Ibid., p. 307.

54. Ibid., p. 296. La partie «Gli uccelli » et sa dernière subdivision «Le ragazze di Soroca » se terminent par cette variante qui conclut le chapitre presque dans les mêmes termes : «"J'ai pitié d'être femme" disse Louise a voce bassa, in quel suo francese di Potsdam. » (Ibid., p. 308)

55. Sur l'attachement de Malaparte à la Laponie, voir C. Malaparte, Les métamorphoses, dans E. Ronchi Suckert (éd.), Malaparte, vol. VI, ouvr. cité, p. 735 : «On me demande souvent d'où vient chez moi ce mépris de la vie en commun avec les hommes, et mon amour, ma passion morbide pour les pays où les hommes sont encore de merveilleux animaux, l'Éthiopie, la Finlande, certains pays de la Russie. Une des raisons qui m'attachent si profondément à la Finlande, cette patrie secrète de mon cœur, c'est l'innocence animale des Finlandais, surtout des Finlandais de Laponie, le merveilleux pays du Nord presque désert. »

56. Relevons à ce propos cet exemple de synthèse originale tirée du journal tenu par Malaparte en Finlande : « La storia del popolo tedesco è una storia tragica: è la storia del contrasto fra quei visi duri e quei corpi nudi, inermi, senza pelle.» (C. Malaparte, Giornale segreto, 2 août, dans E. Ronchi Suckert (éd.), Malaparte, vol. VI, ouvr. cité, p. 251.)

57. «In tutta la letteratura, antica e moderna, intorno alla favolosa "ultima Tule", la renna ricorre non come un elemento della fauna o del paesaggio, voglio dire come un elemento domestico, ma come un elemento morale, come il principale protagonista dei miti, eroici e religiosi. Come la testimonianza di quell'età preistorica, che da lei prende il nome, in cui essa viveva sulle rive del Mediterraneo, in Francia, in Spagna, in Italia.» (C. Malaparte, Viaggio nell'estremo Nord, guerra nell'età della renna, dans E. Ronchi Suckert (éd.), Malaparte, vol. VI, ouvr. cité, p. 207.)

58. Ibid., « Sigfrido e il salmone », p. 341.

59. Ibid., p. 326.

60. " "Sapete, mi dice Dietl, che cosa ho provato la prima volta che son venuto nel Nord? Una specie di vertigine: e insieme il sentimento preciso, la assoluta certezza che avrei potuto sporgere il piede sull'abisso, camminare nel vuoto. [...] Tutto sta nel primo passo, nel posare il piede su quel vuoto, su quell'abisso, su quell'invisibile ponte. È proprio questo ciò che voglio dire; il Nord, questo abisso, questo invisibile ponte sul quale bisogna porre il piede". E dopo un lungo istante di silenzio, aggiunge a bassa voce, guardando fuori dalla finestra: "l'uomo nel Nord, non ha altro appiglio cui aggrapparsi che la propria umanità, che la propria dignità di uomo". » (C. Malaparte, "La dignità umana ", Corriere mercantile, 28 décembre 1942, dans E. Ronchi Suckert (éd.), Malaparte, vol. VI, ouvr. cité, p. 227-228.) 
61. C. Malaparte, Kaputt, p. 338. Le passage reprend l'extrait de l'article "Addio alle renne » (Corriere della sera, 20 avril 1943, dans E. Ronchi Suckert (éd.), Malaparte, vol. VI, ouvr. cité, p. 341-342).

62. «In certe ore del giorno e della notte, quando la luce si ferma, e tutto resta immoto, sospeso sull'abisso immoto dei laghi, delle foreste, dei fiumi, come nei sogni, pareva che il cielo ci avesse abbandonato, che abitassimo una terra senza cielo, che sulle nostre teste si incurvasse il vuoto, il vuoto assoluto, il vuoto dei fisici sperimentali. » (C. Malaparte, Mamma marcia, ouvr. cité, p. 213.) Ce qui montre que le vide du ciel, la nature ennemie de l'homme, le sacrifice de l'animalchristique sont des thèmes inextricablement liés dans l'esprit de Malaparte, c'est qu'après avoir évoqué le caractère abstrait, la solitude du Grand Nord, il évoque à nouveau, comme dans Kaputt, le cimetière des rennes, où il va s'allonger au beau milieu des ossements, tel un soldat antique chanté par Xénophon ou par Eschyle, abandonné parmi les chevaux morts. Ce vide du ciel, le thème de l'homme abandonné par le ciel ne sont pas sans rapport avec celui de l'impossible prière évoqué également dans Mamma marcia. À sa mère mourante qui lui reproche de ne pas prier, le narrateur rappelle qu'en prison, quand il était dans le tourment il ne priait pas non plus mais s'adressait à l'indifférence de Dieu avec violence et avec rage, comme à un ennemi : «Dio se ne infischia dei carcerati [...] Dio non sa che farsene delle nostre preghiere [...] Non gliene importa nulla della vita e della morte di un essere umano. Dio è un egoista. Ed è inutile pregarlo, supplicarlo, buttarsi ai suoi piedi. Se ne infischia.» (Ibid., p. 94-101) Dans un renversement des versets du psaume 107 et du livre de Job récités dans le rituel de Kopparoth (rappelés plus haut dans le cadre de cette étude) où il est dit que celui qui se consume au bord du gouffre peut reprendre espoir et vie grâce à la compassion de Dieu, ici, les anges médiateurs qui apparaissent la nuit dans les prisons sont des « anges malins » (« angeli maligni ») qui au-delà de leur première évocation, marquée d'inquiétante étrangeté, s'avèrent être de tristes punaises, inévitables accompagnatrices nocturnes des prisonniers (ibid., p. 96).

63. Kaputt, « Uomini nudi », p. 333.

64. Ibid., « L'occhio di vetro », p. 258.

65. Ibid., "Golf handicaps », p. 393. Les mouches, animaux-titre du dernier chapitre occupent une place à part dans cette courte liste d'animaux que constituent les principales divisions du roman. Les cinq animaux précédents, qu'ils correspondent à des références analogiques (rats, oiseaux) ou historiques liées à la réalité de la guerre (utilisation des chevaux et des chiens dans les combats) ou géographiques et symboliques (rennes), sont assimilés aux vaincus et aux victimes, alors que les mouches «victorieuses " commencent à envahir les palais romains d'un pouvoir condamné et règnent dans Naples, enfer de chaleur et de pourriture, à la fin du récit. Voir cette très juste remarque du critique Giorgio Barberi Squarotti : «La vittoria delle mosche [...] è il paradosso conclusivo di quella verifica della sconfitta totale della civiltà, degli uomini, della cultura, che Malaparte ha contemplato sui vari fronti, in tutta l'Europa; ed è anche la premessa coerentemente napoletana, dell'atroce viaggio descritto ne La pelle nella degradazione dei vinti, che coinvolge e contamina fino in fondo anche i vincitori. Come è noto, il signore delle mosche è Beelzebub, cioè il nemico dell'uomo, l'irrisore di ogni valore, il trionfatore beffardo sulla corruzione umana che la guerra ha rivelato fino in fondo, nella ferocia delle azioni come nella degradazione interiore. » (G. Barberi Squarotti, L'allegoria degli orrori della guerra, dans R. Barilli et V. Baroncelli (éds), Curzio Malaparte, il narratore, il politologo, il cittadino di Prato e dell'Europa, Naples, Cuen, 2000, p. 291.)

66. Ibid., p. 394. On remarquera que ce passage annonce directement une des thématiques et le titre du roman qui suivra Kaputt, La pelle.

67. Ibid., p. 372.

68. Ibid., p. 4.

69. " "In quale personaggio del tuo dramma si nasconde Cristo?" domandavo a Bulgakov. "Qual è il personaggio che si chiama Cristo?" / "Cristo non ha nome nel mio dramma" rispondeva 
Bulgakov con un tremito di spavento nella voce. "Cristo è un personaggio inutile, ormai, in Russia. Non serve a nulla essere cristiani, in Russia. Non abbiamo più bisogno di Cristo". / "Tu hai paura di dirmi il suo nome", dicevo, "tu hai paura di Cristo". / "Sì, ho paura di Cristo" rispondeva Bulgakov a voce bassa, fissandomi con uno sguardo spaurito. / "Avete tutti paura di Cristo" dicevo a Bulgakov stringendogli forte il braccio. "Perchè avete paura di Cristo?" [...] "Cristo ci odia" diceva Bulgakov a voce bassa, fissandomi con uno sguardo spaurito." (C. Malaparte, Il ballo al Cremlino, Florence, Vallecchi, 1971, p. 68.) Par rapport à ce passage mais aussi par rapport à tout ce dont nous avons traité dans cette étude, l'analyse suivante de Paolo Valesio sur la référence au Christ, nous semble particulièrement juste et éclairante : « Io penso che [...] Malaparte indichi in Cristo uno strumento di scavo della coscienza e di rivelazione del male [...] È chiaro che Malaparte ricorre al Cristo come a una sonda del male in tutti noi. » (P. Valesio, Il dopo-Cristo fra Marinetti e Malaparte, dans R. Barilli et V. Baroncelli (éds), Curzio Malaparte, Il narratore, il politologo, il cittadino di Prato e dell'Europa, ouvr. cité, p. 105.)

70. «Sulla soglia dell'albergo, Majakovskij mi porse la mano, e sorridendo disse a voce bassa: "Maledetti tutti". Io dissi: "Maledetti tutti". Lo seguii con gli occhi mentre si allontanava a passi lenti, alto, elegante, un po' curvo, dondolando le braccia: e mormorai fra i denti "Maledetto anche te". Lo guardavo allontanarsi giù per la via Puscecnaja, e stringevo le labbra per non chiamarlo, per non gridargli di fermarsi, di non andarsene. Avrei voluto corrergli dietro, mettergli una mano sulla spalla, dirgli a voce bassa, affettuosamente: "Maledetto anche Majakovskij”. » (Il ballo al Cremlino, ouvr. cité, p. 97.)

71. C. Malaparte, Mamma marcia, ouvr. cité, p. 136.

72. C. Malaparte, La pelle, ouvr. cité, p. 269.

73. D. Fernandez, Come rendere l'orrore, la lezione di Malaparte, dans M. Grassi (éd.), «La bourse des idées du monde». Malaparte e la Francia, Atti del convegno internazionale di studi su Curzio Malaparte (Prato-Florence, 2007), Florence, Olschki, 2008, p. 7.

74. G. Bàrberi Squarotti, L'allegoria degli orrori della guerra, dans R. Barilli et V. Baroncelli (éds), Curzio Malaparte il narratore, il politologo, il cittadino di Prato e dell'Europa, ouvr. cité, p. 301.

75. C. Malaparte, Les métamorphoses, dans E. Ronchi Suckert (éd.), Malaparte, vol. VI, ouvr. cité, p. 732-733. L'allusion à la modification des cordes vocales des animaux ne peut pas ne pas faire résonnance avec la mort «fantasmée » du chien Febo dans La pelle, à qui on a coupé les cordes vocales, comme à ses congénères, pour ne pas les entendre geindre pendant les expérimentations de la clinique vétérinaire.

76. Ibid., p. 741.

77. «Temps des fables » : expression que l'on retrouve d'ailleurs dans la citation de Montesquieu qui conclut le texte de Malaparte introductif à Kaputt, p. 4.

78. "Toute violence désormais révèle ce que révèle la passion du Christ, la genèse imbécile des idoles sanglantes, de tous les faux dieux des religions, des politiques, des idéologies. » (R. Girard, Le bouc émissaire, Paris, Grasset, 1982, p. 295)

\section{RÉSUMÉS}

Dans le récit Kaputt évoquant les horreurs du conflit mondial, le stylème "à voix basse » accompagne de façon récurrente les déclarations du narrateur, témoin privilégié en tant qu'envoyé spécial suivant l'armée allemande dans le nord de l'Europe, mais aussi les paroles des 
victimes et des bourreaux conquérants. L'étude vise à montrer que cette expression, relevant du secret, de la menace et de l'effroi mais aussi de l'interdit, de la malédiction et de la révélation est intimement liée à la mystérieuse division du roman en noms d'animaux et au thème du sacrifice qui prend, dans cette étape de l'œuvre malapartienne, une nouvelle ampleur.

Nel romanzo Kaputt, sugli orrori del secondo conflitto mondiale, lo stilema «a voce bassa» accompagna in modo ricorrente le dichiarazioni del narratore, testimone privilegiato in quanto inviato speciale al seguito dell'esercito tedesco nell'Europa del Nord, ma anche le parole delle vittime e dei carnefici conquistatori. Lo studio intende mostrare che questa espressione, appartenente all'ambito del segreto, della minaccia e dello spavento, ma anche del divieto, della maledizione e della rivelazione, è intimamente legata alla misteriosa divisione del romanzo in nomi di animali e al tema del sacrificio che prende una nuova ampiezza in questa tappa dell'opera di Malaparte.

In Kaputt, a novel dealing with the horrors of the Second World War, the phrase "in a low voice" is recurrently attached to the narrator's statements-as a special correspondent following the German army in northern Europe, the latter is a privileged observer of the conflict-but also to the words both of the victims and of the conquering persecutors. This essay is aimed at showing how the phrase-which conveys the notions of secrecy, threat and fright as well as interdiction, malediction and revelation-is actually closely linked with the mysterious division of the novel into chapters whose titles refer to animals and with the topic of sacrifice which, in this part of Malaparte's oeuvre, is given a new dimension.

\section{INDEX}

Keywords : low voice, Hell, Inferno, far North, Nazis, horses, crucifixion, sacrifice, victim

Parole chiave : voce bassa, inferno, Gran Nord, nazisti, cavalli, crocifissione, sacrificio, vittima

Mots-clés : voix basse, enfer, Grand Nord, nazis, chevaux, crucifixion, sacrifice, victime

\section{AUTEUR}

\section{MICHĖLE COURY}

Université Grenoble Alpes 